\title{
Positron Lifetimes in Pure and Doped Ice and in Water
}

\author{
Eldrup, Morten Mostgaard; Mogensen, O.; Trumpy, Georg
}

Published in:

Journal of Chemical Physics

Link to article, DOI:

$10.1063 / 1.1677990$

Publication date:

1972

Document Version

Publisher's PDF, also known as Version of record

Link back to DTU Orbit

Citation (APA):

Eldrup, M. M., Mogensen, O., \& Trumpy, G. (1972). Positron Lifetimes in Pure and Doped Ice and in Water. Journal of Chemical Physics, 57(1), 495-504. https://doi.org/10.1063/1.1677990

\section{General rights}

Copyright and moral rights for the publications made accessible in the public portal are retained by the authors and/or other copyright owners and it is a condition of accessing publications that users recognise and abide by the legal requirements associated with these rights.

- Users may download and print one copy of any publication from the public portal for the purpose of private study or research.

- You may not further distribute the material or use it for any profit-making activity or commercial gain

- You may freely distribute the URL identifying the publication in the public portal

If you believe that this document breaches copyright please contact us providing details, and we will remove access to the work immediately and investigate your claim. 


\title{
Positron Lifetimes in Pure and Doped Ice and in Water
}

\author{
M. Eldrup ANd O. Mogensen \\ Chemistry Department, Danish Atomic Energy Commission Research Establishment, Risö, Roskilde, Denmark \\ AND \\ G. TRUMPY \\ Laboratory of A pplied Physics II, Technical University of Denmark, Lyngby, Denmark
}

(Received 8 February 1972)

\begin{abstract}
Positron lifetime spectra were measured in mono- and polycrystalline light ice, polycrystalline heavy ice, doped light ice, as well as in light and heavy water. All spectra were resolved into three components. At temperatures between $-196^{\circ}$ and $-100^{\circ} \mathrm{C}$ the lifetimes and relative intensities are virtually constant, being $0.12 \mathrm{nsec}$ and $20 \%, 0.44 \mathrm{nsec}$ and $28 \%, 0.68 \mathrm{nsec}$ and $52 \%$, and attributed to annihilation of para-Ps, free positrons, and ortho-Ps, respectively. Above $-100^{\circ} \mathrm{C}$ the longest lifetime increases with temperature to $1.1 \mathrm{nsec}$ at $0^{\circ} \mathrm{C}$. Its relative intensity is unchanged, while the parameters for the other components show a complex behavior. The spectra for mono- and polycrystalline light ice and for polycrystalline heavy ice are identical. For water long lifetime components attributed to ortho-Ps are $1.86 \mathrm{nsec}, 27 \%$ for $\mathrm{H}_{2} \mathrm{O}$ and $2.01 \mathrm{nsec}, 22 \%$ for $\mathrm{D}_{2} \mathrm{O}$. Theoretical explanations are suggested. Fast frozen solutions of $\mathrm{HF}$ with concentrations larger than approximately $10^{-5}$ mole fraction have a lifetime component at $-160^{\circ} \mathrm{C}$ of 1.23 nsec, $52 \%$ attributed to trapping of ortho-Ps in amorphous regions. Very good agreement is found between the experiments and a trapping model. Irreversible changes of the spectra are found by heating above approximately $-120^{\circ} \mathrm{C}$. Measurements on a number of fast frozen aqueous solutions of acids, bases, and salts are reported, none of them showing as strong influence on the ortho-Ps lifetime as HF.
\end{abstract}

\section{INTRODUCTION}

A positron entering a solid is nearly always thermalized before annihilating with an electron into photons. The photon properties depend on the electron-positron state on annihilation. Hence experimental studies of the annihilation photons ${ }^{1,2}$ may give useful information about the structure of the solid. In particular the interaction of positrons with defects has been a subject of interest for some years past. Recent experiments have indicated that for defect concentration above approximately the parts per million level, the positrons may become trapped in the defects (e.g., vacancies in metals, ${ }^{3,4} \mathrm{~F}$ centers, and cation vacancies in ionic crystals $^{5,6}$ ) before annihilation.

Two experimental methods ${ }^{1}$ are mainly used in positron annihilation research: the positron lifetime technique (see next section) and the angular correlation technique. The two photons in the $2 \gamma$ annihilation are emitted in nearly opposite directions. An angular correlation setup determines the distribution of photon pairs as function of the angle $\theta$, where $\pi-\theta$ is the angle between the wave vectors of the two photons. In a rough first approximation $\theta$ is proportional to the center-of-mass momentum of the electron-positron pair.

In ice about $70 \%$ of the positrons form a hydrogenlike atom, positronium (Ps: $\frac{1}{4}$ para-Ps and $\frac{3}{4}$ ortho- $\mathrm{Ps}_{\mathrm{s}}$ ) before annihilation. The positrons will therefore in ice mainly decay in three different ways: (1) by "free" annihilation with the outer electrons of the molecules (intensity $I \simeq 30 \%$, lifetime $\tau \simeq 0.4 \mathrm{nsec}$, gives a broad angular correlation curve); (2) by ortho-Ps pickoff annihilation, i.e., the positron annihilates with an outer electron of the molecules during an ortho-Ps molecule collision $(I \simeq 52 \%, \tau=0.7-1.3$ nsec, gives a broad angular correlation curve); and (3) by para-Ps intrinsic annihilation $(I \simeq 18 \%, \tau \simeq 0.12 \mathrm{nsec}$; some para-Ps pickoff annihilation is also found). A narrow central and narrow sidepeaks in the angular correlation curves ${ }^{7}$ for single crystals of ice at low temperatures have been interpreted as due to intrinsic para-Ps annihilation from a center-of-mass Bloch function state, i.e., para-Ps (and hence also ortho-Ps) is delocalized on annihilation. Para-Ps trapped in defects will give a somewhat broader central component of the angular correlation curve due to a high zero-point energy in the trap. The sidepeaks disappear on trapping. Because of reduced pickoff annihilation, trapping of Ps increases the ortho-Ps lifetime.

In this Article we discuss the results of positron lifetime studies of the motion of Ps in ice and doped ice. We have found that Ps is trapped in defects at higher temperatures and for high enough concentrations of impurities (mainly HF doping has been studied). The work is part of an experimental series on positron annihilation in ice and doped ice. In Ref. 8 we showed that a change in ortho-Ps lifetime and yield takes place at the melting point for both light and heavy water. Reference 7 established the fact that Ps is delocalized in ice at low temperatures. Angular correlation curves for frozen aqueous solutions were published in Ref. 9. Work on angular correlation as function of temperature in pure ice, ${ }^{10}$ on angular correlation and lifetime studies of HF-doped ice single crystals, and on angular correlation magnetic quenching in ice ${ }^{11}$ are in progress.

\section{EXPERIMENTAL METHODS}

A ${ }^{22} \mathrm{Na}$ positron source emits a $1.27 \mathrm{MeV}$ photon nearly simultaneously with the emission of the positron. The positron lifetime is determined as the difference in time between detection of the $1.27 \mathrm{MeV}$ photon and 
one of the $0.51 \mathrm{MeV}$ annihilation photons. The measured distribution of lifetimes is known as the lifetime spectrum. Each annihilation process contributes to the spectrum a decaying exponential, its decay constant being the inverse of the mean lifetime.

For the measurements we used a conventional ${ }^{1}$ fast-slow delayed-coincidence system mainly consisting of Ortec electronic units and a Nuclear Data 2200 multichannel analyzer with 4096 channels of which 512 were used for recording a spectrum. The width of the prompt curve was 0.425 nsec FWHM. As a source ${ }^{22} \mathrm{NaCl}$, of approximately $50 \mu \mathrm{Ci}$ sandwiched between two foils of Mylar, was used. The polycrystalline ice samples were prepared by freezing the water in a cylindrical brass container of dimensions $10 \mathrm{~mm}$ diameter and $15 \mathrm{~mm}$ height. Before freezing, the source was inserted as a plane through the cylinder axis, so that it would be lying between two half-cylinders of ice. The light water used for the polycrystalline samples was distilled twice. Its specific conductivity was approximately $10^{-6} \mathrm{ohm}^{-1} \cdot \mathrm{cm}^{-1}$ and it contained an equilibrium amount of air. In order to investigate the possible influence of dissolved oxygen, samples were prepared containing no dissolved oxygen. However, within the experimental uncertainties no difference was found between these samples and ordinarily prepared ones. The heavy water had a specific conductivity of less than $10^{-5} \mathrm{ohm}^{-1} \cdot \mathrm{cm}^{-1}$ before freezing. The monocrystalline samples were cut from a larger monocrystal to dimensions $10 \times 10 \times 4 \mathrm{~mm}^{3}$. After being ground and polished two such pieces of crystal were assembled in a sample container with the source between them.

The frozen solutions were prepared by immersing the brass container with the solution in liquid nitrogen. The container reached liquid nitrogen temperature in about $1 \mathrm{~min}$. This way of cooling will be referred to as "fast freezing." All spectra have been corrected for annihilation in the source, which amounted to $7 \%$ to $10 \%$ of the total number of annihilations. ${ }^{12}$

\section{DATA ANALYSIS}

The lifetime spectra were analyzed by use of the leastsquares fitting computer program POSITRONFIT described in Ref. 12. In principle it fits to an experimental spectrum a curve composed of a sum of decaying exponentials and a constant background. The results of the fitting procedure are the reciprocal decay constant (i.e., lifetime) and the relative intensity of each component (i.e., the area under each exponential in percent of the whole area). The goodness of the fit is expressed by the quantity, the "variance of the fit." It is essentially a normalized sum of the squares of the deviations between the fitted curve and the measured spectrum. For a fit to be satisfactory, the variance of the fit should be close to unity, since perfect fits will give variances which have a normal distribution, with a mean value of unity and with a standard deviation which for the present measurements is 0.072 . A large deviation from unity suggests that the resulting parameters are not a good representation of the measured spectrum.

One of the experiences gained from the analysis of our measurements was that all spectra could be fitted completely by three exponential terms, i.e., the variance of a fit including four terms was nearly the same as that for three terms. On the other hand a two-term fit was always appreciably poorer than a three-term fit except for a few special cases.

These facts do not necessarily mean that all spectra contained no more than three lifetime components, but only that the number of fitting parameters were sufficient to give a complete representation of a measured spectrum. If a spectrum is composed of more than three components the question then arises as to the physical significance of the parameters obtained from an analysis. Strictly speaking they are only mathematical numbers describing a curve which is a good approximation to the spectrum. If in an analysis weight is attached to one part of the spectrum, and in another analysis of the same spectrum to another part, the parameters resulting from the analyses are likely to be different in the two cases. The weight can be changed for example by changing the numerical weight ascribed to each point in a least-sqares fitting procedure or by imposing some sort of constraints on the parameters to be fitted. An often used method of analysis is to determine the component with the longest lifetime and subtract it from the spectrum. The difference is then considered as an unresolvable mixture of shorter lifetimes. This method attaches great weight to the long-lived component and more or less ignores the detailed shapes of the short-lived ones.

If the longest lifetime is appreciably longer (i.e., two to three times or more) than the shorter ones and has an intensity of at least a few percent, it is so well defined that all reasonable methods of analysis should give the same result for this component, while the results for the shorter-living components may differ (especially when they are of the order of magnitude of the equipment time resolution). However, if the various lifetimes in a spectrum are rather close in magnitude, it may be difficult to extract directly well-defined parameters. During the analysis it may then be necessary to use knowledge about some of the parameters gained from other measurements. The results presented below for water and pure ice are examples of those two different situations.

In general it cannot be said what particular method of analysis should be used for a spectrum which contain more than a resolvable number of components. The only requirement is that the resulting parameters must represent a curve that fits the measured spectrum reasonably well. The method to be chosen for a certain problem should be the one which most efficiently extract the wanted physical information from the spectrum. 
TABLE I. Lifetimes and relative intensities for $\mathrm{H}_{2} \mathrm{O}$ and $\mathrm{D}_{2} \mathrm{O}$ at $20^{\circ} \mathrm{C}$. The uncertainties are standard deviations as estimated by the computer.

\begin{tabular}{ccccccc}
\hline & $\begin{array}{c}\tau_{1} \pm \Delta \tau_{1} \\
(\mathrm{nsec})\end{array}$ & $\begin{array}{c}\tau_{2} \pm \Delta \tau_{2} \\
(\mathrm{nsec})\end{array}$ & $\begin{array}{c}\tau_{3} \pm \Delta \tau_{3} \\
(\mathrm{nsec})\end{array}$ & $\begin{array}{c}I_{1} \pm \Delta I_{1} \\
(\%)\end{array}$ & $\begin{array}{c}I_{2} \pm \Delta I_{2} \\
(\%)\end{array}$ & $\begin{array}{c}I_{3} \pm \Delta I_{3} \\
(\%)\end{array}$ \\
\hline $\mathrm{H}_{2} \mathrm{O}$ & $0.22 \pm 0.02$ & $0.46 \pm 0.02$ & $1.86 \pm 0.02$ & $25.0 \pm 5.1$ & $48.2 \pm 4.7$ & $26.9 \pm 0.5$ \\
$\mathrm{D}_{2} \mathrm{O}$ & $0.31 \pm 0.03$ & $0.53 \pm 0.06$ & $2.01 \pm 0.03$ & $44.6 \pm 12.6$ & $33.2 \pm 12.1$ & $22.2 \pm 0.6$ \\
\hline
\end{tabular}

The problems involved in the analysis of positron lifetime spectra have only been discussed to a rather small extent in the literature. The above considerations and the following discussion of the analysis of our data show, however, that these problems deserve more attention than has usually been paid to them.

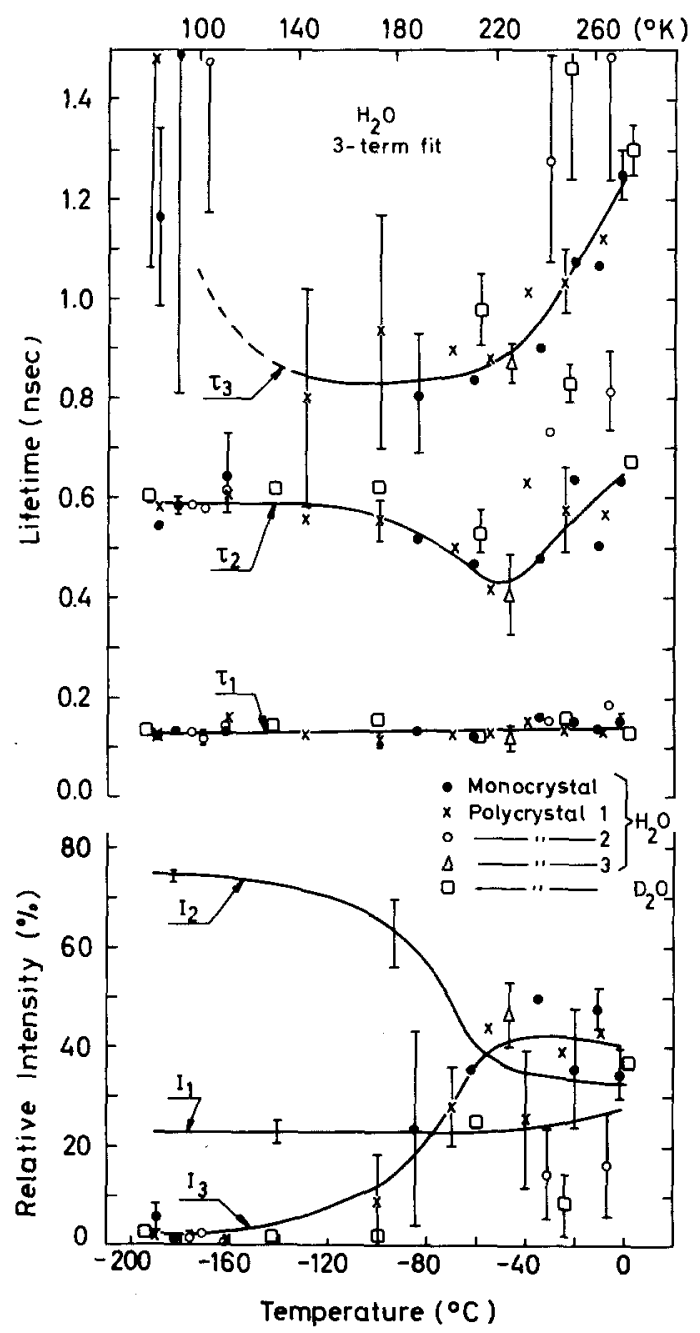

FIc. 1. Lifetimes and relative intensities for a three-term analysis of lifetime spectra for light and heavy ice as function of temperature. The experimental points for $I_{1}$ and $I_{2}$ have been omitted for the sake of clarity. Typical uncertainties indicated on some of the experimental points are standard deviations as estimated by the computer.

\section{EXPERIMENTAL RESULTS}

\section{A. Pure Water and Ice}

The results published in Ref. 8 demonstrated that a discontinuity exists in the lifetime and relative intensity of ortho-Ps at the ice-water phase transition. In order to be able to use the more refined method of analysis provided by POSITRONFIT, ${ }^{12}$ the spectra were remeasured and the temperature region extended down to liquid nitrogen temperature. Within the experimental uncertainties the results of the analysis were in agreement with Ref. 8. The results for light and heavy water at $20^{\circ} \mathrm{C}$ are given in Table $\mathrm{I}$.

Here and in the following the lifetimes and relative intensities are numbered from shortest to longest lifetime. This is done since no convention is commonly used for results analyzed in terms of more than two lifetimes.

The results for $\mathrm{H}_{2} \mathrm{O}$ and $\mathrm{D}_{2} \mathrm{O}$ ice as analyzed by a three-term fit are shown in Fig. 1. The standard deviations as estimated by the computer are given as vertical bars on some of the experimental points. For the sake of clarity the points for $I_{1}$ and $I_{2}$ have been omitted, and only typical uncertainties have been indicated. Furthermore, a few points with extremely large uncertainties have been omitted. Note that large uncertainties in this case indicate that a good fit to the experimental data can be obtained within a wide range of the parameters. Thus, the points shown in Fig. 1 reproduce the data extremely well.

It is seen that particularly the short lifetime $\tau_{1} \simeq 0.12$ nsec and its intensity $I_{1}$ are well-defined quantities by this procedure. As discussed earlier, one can obtain a better determination of parameters and a possibility for physically more meaningful results by introducing constraints, reducing the number of free parameters from essentially five (three lifetimes and two relative intensities) to essentially three or four (for details about the parameters used in the fitting procedure, see Ref. 12).

In Fig. 2 is shown a two-term fit (three parameters) for the same set of data as above. It is seen that the data for $\mathrm{H}_{2} \mathrm{O}$ and $\mathrm{D}_{2} \mathrm{O}$ agree closely. The curves in Fig. 2 naturally contain essential information about the experimental data, but they do not provide a complete reproduction of the experimental data as is shown by the difference $\Delta_{2,3}$ between the variances of the two- 

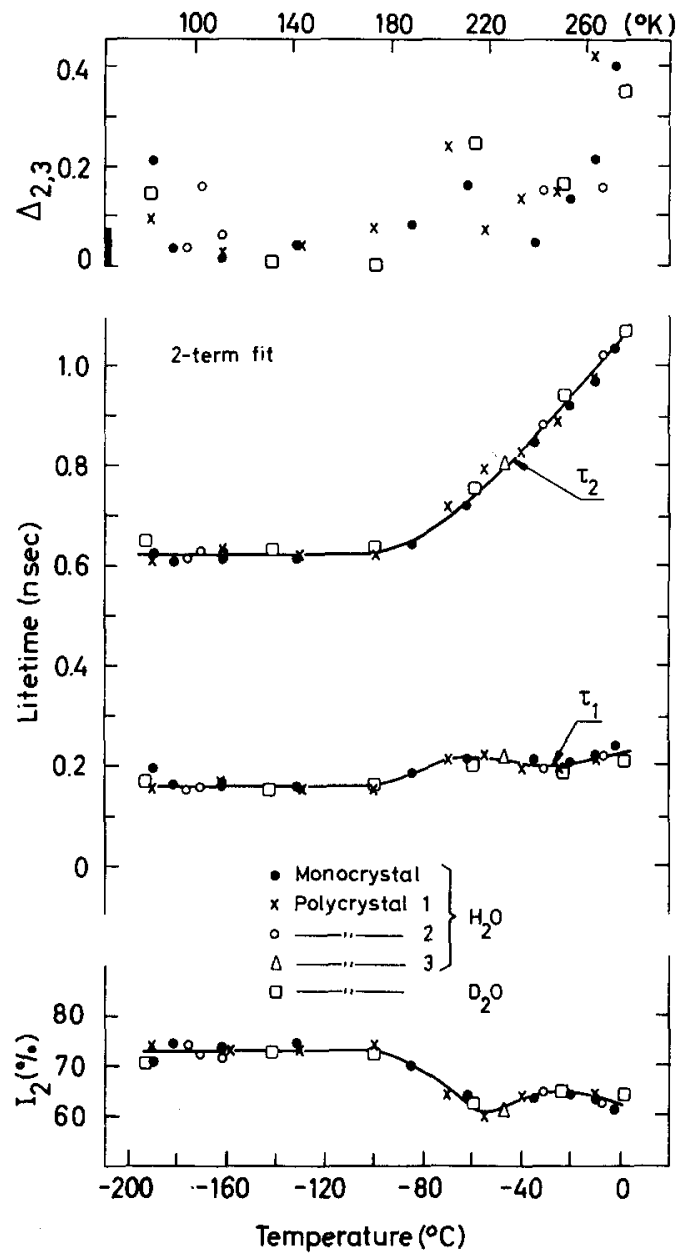

FIG. 2. Lifetimes and relative intensities for a two-term analysis of lifetime spectra for light and heavy ice as function of temperature. $\Delta_{2,3}$ indicates the difference between the variance of a two-term and the corresponding three-term fit. The statistically determined standard deviation for the variance is indicated by a black bar.

term and the corresponding three-term fits. We mentioned earlier that the variance of a three-term fit is the smallest that can be obtained for a particular spectrum. The value of the variance for various spectra is subject to statistical fluctuations. Since these fluctuations are almost eliminated in $\Delta_{2,3}$, this quantity more directly shows the goodness of a two-term fit than does the variance of the fit itself. As the values of $\Delta_{2,3}$ in Fig. 2, except for a few points, are of the order of or larger than the standard deviation of the variance (shown by the black bar), the two-term fits must be considered rather poor.

In order to obtain a physically more useful result we now try a four-parameter fit under the assumption that $I_{3}$ is the ortho-Ps annhilation intensity and that it is independent of temperature. This assumption is based upon the following considerations:

1. The Ps-formation probability is expected to depend only slightly on temperature from $0^{\circ} \mathrm{C}$ and down, since the energies involved in temperature changes are of the order of $1 \%$ or $2 \%$ of an electron volt, while energies involved in determination of the Ore gap are of the order of 1 to $10 \mathrm{eV} .^{13}$

2. From angular correlation measurements one finds that the total relative area of narrow components associated with para-Ps is equal to $15.2 \pm 0.5 \%$ for temperatures between -182 and $0^{\circ} \mathrm{C} .{ }^{7}, 10$ Taking the pickoff process into account a total para-Ps intensity of $18 \pm 0.5 \%$ is found. This entails an ortho-Ps intensity of $54 \pm 1.5 \%$, if the reasonable assumption is made that no ortho-para conversion takes place.

3. In the measurements on doped ice described below we find for the higher $\mathrm{HF}$ concentrations a long-lived component of intensity $52 \pm 2 \%$ at $-160^{\circ} \mathrm{C}$. It may reasonably be ascribed to ortho-Ps. When these measurements are performed as a function of temperature, the intensity of the long-lived component stays roughly constant, varying between $42 \%$ and $54 \%$ in the interval from -196 to $-20^{\circ} \mathrm{C}$.

4. The $3 \gamma$-annihilation rate is, to a good approximation, proportional to the product of the ortho-Ps lifetime and its intensity ${ }^{1}$ :

$$
R_{3 \gamma} \propto I_{\text {ortho }} \cdot \tau_{\text {ortho }} .
$$

In Fig. 3 the measurements of Wagner and Hereford ${ }^{14}$ of the $3 \gamma$ rate are compared with the products of the longest lifetimes and their corresponding intensity as

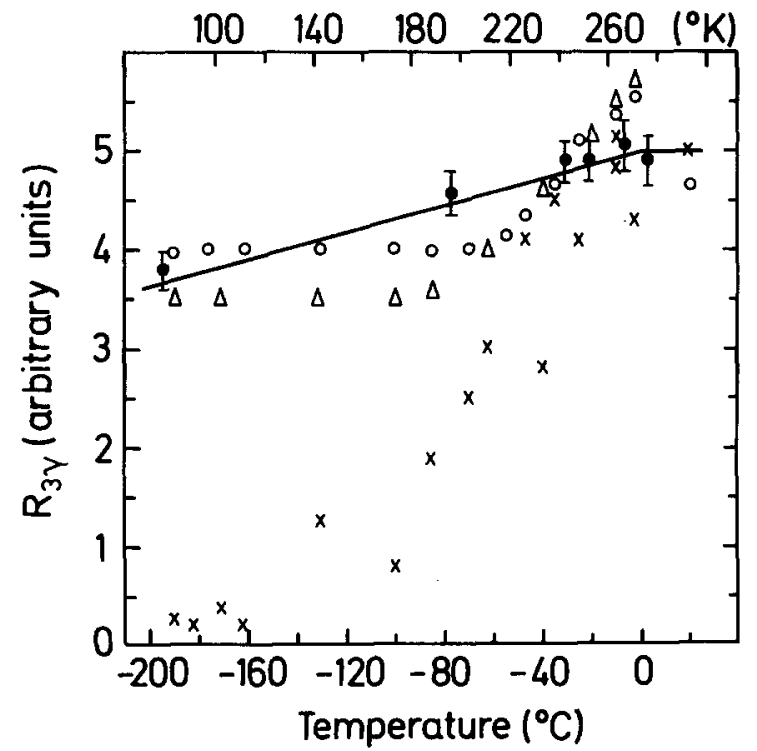

FIG. 3. Rate of $3 \gamma$ annihilations in ice, as obtained by Wagner and Hereford, ${ }^{14}$ compared with the product of the longest lifetime and its relative intensity from the present measurements. Results are shown for different fitting procedures. The ordinate has been normalized to give the best visual fit to the results of Wagner and Hereford. (with vertical error bars): Wagner and Hereford; $O$ : two-term fit; $X$ : three-term fit; $\triangle$ : three-term fit, $I_{3}=52 \%$. 
determined by two- and three-term fits of the present measurements. It is evident that the variation of $I_{\text {ortho }}$ with temperature must be much smaller than of $I_{3}$ as obtained by the first three-term fit.

These points, taken together, lead us to the conclusion that the total amount of ortho-Ps formed in ice must be $52 \pm 2 \%$, varying only little with temperature. Accordingly, the lifetime spectra have finally been fitted by use of three lifetime terms, but subject to the constraint that $I_{3}=I_{\text {ortho }}=52 \%$ (four-parameter fit). This implies that $\tau_{3}$ is now assumed to be an average ortho$\mathrm{Ps}$ lifetime. The computed results for $\mathrm{H}_{2} \mathrm{O}$ are shown in Fig. 4. In this case the lifetime spectra are quite well represented by the obtained parameter set, as shown by the small differences $\Delta_{3}{ }^{\prime}, 3$ between the variance of the four-parameter three-term fit and the five-parameter
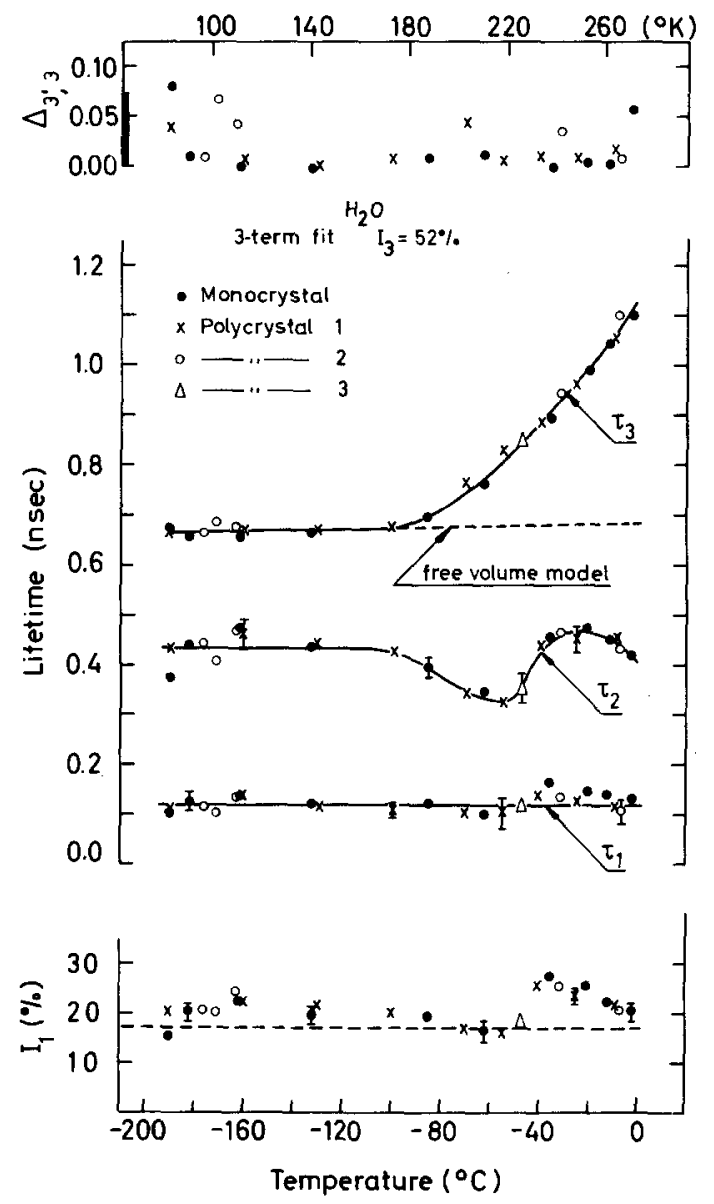

FIG. 4. Lifetimes and relative intensities for a three-term analysis of lifetime spectra for light ice subject to the constraint $I_{3}=52 \%$. The indicated typical uncertainties are the standard deviations as estimated by the computer. $\Delta_{3^{\prime}, 3}$ is the difference between the variance of this analysis and the corresponding three-term fit without constraints. The statistically determined standard deviation for the variance is indicated by the black bar. The calculated curve for the free volume model is fitted to experiments at $-190^{\circ} \mathrm{C}$. three-term fit. These differences are generally considerably smaller than the standard deviation of the variance.

Figure 4 shows that the ortho-Ps lifetime is nearly constant (around $0.68 \mathrm{nsec}$ ) for temperatures below $-100^{\circ} \mathrm{C}$. Above $-100^{\circ} \mathrm{C}$ it increases with increasing temperature. Furthermore, we note that the results for mono- and polycrystalline $\mathrm{H}_{2} \mathrm{O}$ agree closely. The obtained product $I_{3} \tau_{3}$ has been plotted in Fig. 3 for comparison with $R_{3 \gamma}$. The temperature dependence are similar but not quite identical.

One may naturally ask why the results in Fig. 1 are so different from those of Fig. 4. The main reason probably is that the analyzed spectra contain more than three exponential terms, even when corrected for source annihilation. As seen in Fig. 1 a long-lived component of a relative intensity of $1 \%$ or $2 \%$ is resolved at low temperatures. The influence of this component on the fitted parameters is greatly reduced in the two-term fit and in the three-term fit with the intensity $I_{3}$ fixed at $52 \%$. However, it strongly influences the results of the three-term fit without constraints. The origin of this component may be of various natures. It may be an inherent part of a true ice spectrum. It may be caused by small uncontrolled amounts of impurities (the deviating results of polycrystal run 2 in Fig. 1 are probably caused by this), or it may be caused by insufficient source correction. The uncertainties involved in the correction procedures are discussed in Ref. 15.

\section{B. Frozen Solutions of HF}

In many areas of ice physics, ice is doped with hydrogen fluoride and ammonia in order to create defects (so-called $\mathrm{L}$ and $\mathrm{D}$ defects). ${ }^{16-18}$ With the purpose of investigating the influence of HF doping on the lifetime of positrons, aqueous solutions of different $\mathrm{HF}$ concentrations were fast frozen, and measurements were made at $-160^{\circ} \mathrm{C}$.

We found that a long-lived component appeared in the spectrum even for rather small $\mathrm{HF}$ concentrations. For concentrations above $5 \times 10^{-5}$ mole fraction its lifetime and relative intensity as determined from several spectra were $1.23 \pm 0.02 \mathrm{nsec}$ and $52 \pm 2 \%$. As is known from angular correlation results (see previous paragraph) there is good reason to assume that orthoPs is formed with a relative yield close to $52 \%$ in pure ice. Accordingly we interpret the increased lifetime as due to ortho-Ps trapped in HF-created defects in which the lifetime against pickoff is $1.23 \mathrm{nsec}$.

A simple model has been used to describe the trapping of positrons. ${ }^{3,5}$ According to the model, positrons in the bulk will either annihilate (with rate $\lambda_{b}$ ) or become trapped in defects (rate $\kappa$, proportional to defect concentration). A positron in a trap will annihilate with the rate $\lambda_{t}\left(=1 / \tau_{t}\right)$. The model shows that a lifetime spectrum for such positrons will have two components, 


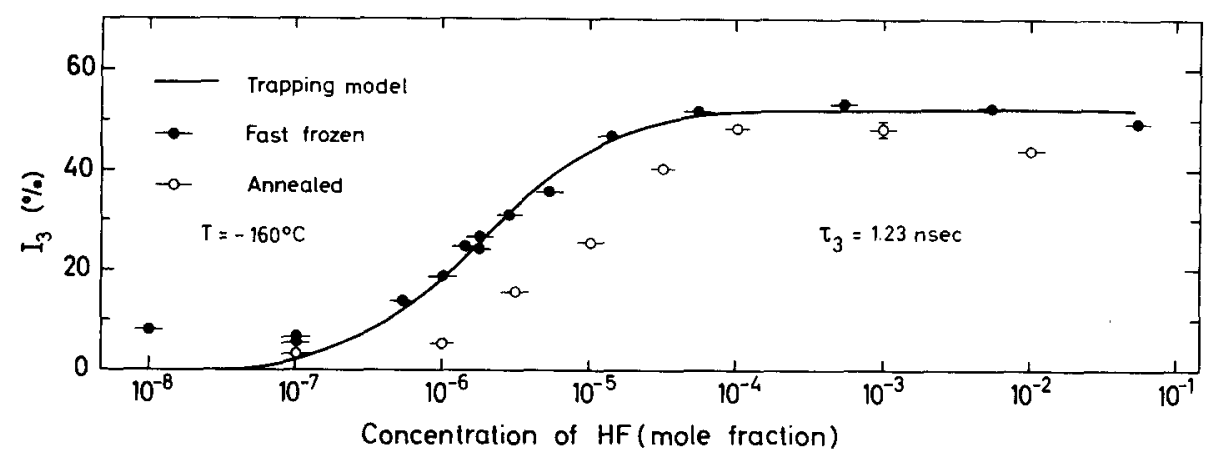

FIG. 5. Relative intensity of the component with lifetime $1.23 \mathrm{nsec}$ as function of $\mathrm{HF}$ concentration for fast frozen samples, and for fast frozen samples that were annealed for $20 \mathrm{~h}$ at $-18^{\circ} \mathrm{C}$ and subsequently cooled at the rate of about $1^{\circ} \mathrm{C} / \mathrm{min}$. The results are compared with the trapping model, Eq. (1). one with the decay rate $\lambda_{b}+\kappa$ and one with the decay rate $\lambda_{l}$. The relative intensity of the latter component is

$$
I_{t}=I_{0} \kappa /\left(\lambda_{b}-\lambda_{t}+\kappa\right)
$$

$I_{0}$ being the total intensity. By applying this model to trapping of ortho-Ps we analyzed the measured spectra by three-term fits, keeping one lifetime fixed at 1.23 nsec. (This is another case, where results obtained from other measurements are used to extract physical information from lifetime spectra which contain more than three lifetime components). The intensity of the component with lifetime $1.23 \mathrm{nsec}$ as a function of $\mathrm{HF}$ concentration is compared with the trapping model curve in Fig. 5 ( $\kappa$ assumed proportional to HF concentration, $I_{0}=52 \%$ ). Furthermore the figure shows the results for samples that after fast freezing were annealed for $20 \mathrm{~h}$ at $-18^{\circ} \mathrm{C}$ and then cooled to $-160^{\circ} \mathrm{C}$ with the rate of $1^{\circ} \mathrm{C}$ per min.

In order to further investigate the transition from the fast frozen to the annealed state, samples were heated to different temperatures. Then they were again cooled to $-160^{\circ} \mathrm{C}$ and their lifetime spectra recorded. We found that the transition takes place below $-100^{\circ} \mathrm{C}$ since no

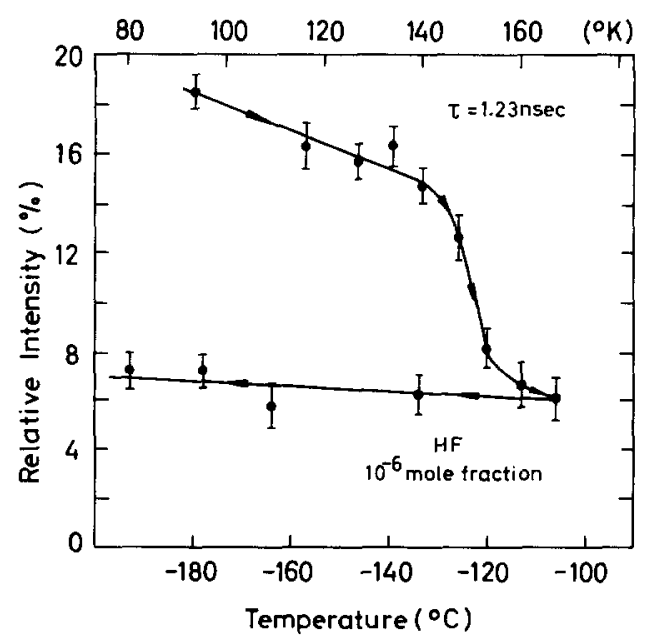

Frg. 6. Transition from fast frozen state to annealed state as indicated by the relative intensity of the component with lifetime 1.23 nsec. The interval between subsequent measurements were 1 day, the cooling rate approximately $1^{\circ} \mathrm{C} / \mathrm{min}$. further change of the spectra occurred by heating the samples to higher temperatures. The temperature region up to $-100^{\circ} \mathrm{C}$ was subsequently investigated in more detail for a $\mathrm{HF}$ concentration of $10^{-6}$ mole fraction. The intensity of the $1.23 \mathrm{nsec}$ component as function of temperature is shown in Fig. 6. An irreversible transition is clearly seen to take place between approximately -130 and $-120^{\circ} \mathrm{C}$.

\section{Other Frozen Solutions}

The main difference between the spectra for frozen solutions and that for pure ice (all at $-160^{\circ} \mathrm{C}$ ) was the appearance of a long-lived component of an intensity varying from a few percent to around 50\% depending on solution and concentration. To get an approximate quantitative expression for the intensity of this longlived component all spectra were fitted with three terms, one lifetime being fixed at 1.23 nsec. The intensity of this component gives the wanted information.

Frozen $\mathrm{NH}_{3}$-solution measurements were made also at other temperatures. Only small differences from the results of pure ice were seen. The most important results are given in Table II.

A number of other fast frozen solutions of salts and acids in ice were investigated at one or two different concentrations in order to compare with the $\mathrm{HF}$ and $\mathrm{NH}_{3}$ results. None of them influenced the lifetime as strongly as $\mathrm{HF}$. The results are listed in Table III.

TABLE II. Relative intensities of component with lifetime $1.23 \mathrm{nsec}$ at $-160^{\circ} \mathrm{C}$ and lifetime and intensity of long-lived component at $-9^{\circ} \mathrm{C}$ for frozen aqueous solutions of $\mathrm{NH}_{3}$. The last three rows were obtained in chronological order from above to below. The uncertainties are standard deviations as estimated by the computer.

\begin{tabular}{ccccc}
\hline \hline $\begin{array}{c}\text { Concentra- } \\
\text { tion (mole } \\
\text { fraction) }\end{array}$ & $\begin{array}{c}\text { Tempera- } \\
\text { ture } \\
\left({ }^{\circ} \mathrm{C}\right)\end{array}$ & $\begin{array}{c}\tau_{3} \pm \Delta \tau_{3} \\
(\mathrm{nsec})\end{array}$ & $\begin{array}{c}I_{3} \pm \Delta I_{3} \\
(\%)\end{array}$ & Comment \\
\hline $4 \times 10^{-2}$ & -160 & 1.23 & $9.1 \pm 0.9$ fast frozen \\
$10^{-3}$ & -160 & 1.23 & $5.0 \pm 0.1$ fast frozen \\
$10^{-3}$ & -9 & $1.22 \pm 0.07$ & $34.5 \pm 7.6$ \\
$10^{-3}$ & -160 & 1.23 & $5.7 \pm 0.5$ annealed \\
\hline \hline
\end{tabular}




\section{DISCUSSION}

\section{A. Water and the Ice-Water Transition}

The light water values for the long lifetime and its relative intensity agree very well with most of the published data on these quantities. ${ }^{1}$ This component is attributed to annihilation of ortho-Ps. The relative intensity also agrees with the value of $36 \pm 1 \%$ for the total Ps formation as determined by angular correlation magnetic quenching experiments. ${ }^{11}$

When going from the solid to the liquid phase there is a considerable increase in the long lifetime. Also for a few other molecular materials values of the long lifetime in both solid and liquid phase have been published. Generally the long lifetime is longer in the liquid than in the solid. ${ }^{13,19,20}$ For some substances the change has been correlated to a change in "free volume" (i.e., the volume not occupied by the molecules) in agreement with the "free volume model", ${ }^{13,21}$ but in a few other cases this is not possible ${ }^{22}$ and the lifetime in the liquid is longer than predicted by the volume change. Water is an example of this. ${ }^{8,21}$

Ps is able by exchange forces to create a bubble around itself in a liquid. This fact has been used to explain the very long lifetimes (tens of nanoseconds) observed in some liquified gases, ${ }^{2}$ and has also been applied more or less directly to ordinary liquids. ${ }^{2,23,24}$ Because of the reduced electron density in the bubble the ortho-Ps lifetime against pickoff annihilation will be longer than could be expected from the average electron density of the liquid. It is likely that bubble formation is responsible for the general increase in ortho-Ps lifetime in liquids compared to solids as mentioned above.

Another noteworthy fact is the difference between liquid $\mathrm{H}_{2} \mathrm{O}$ and $\mathrm{D}_{2} \mathrm{O}$ when one considers the long life-

TABLE III. Relative intensity of component with lifetime $1.23 \mathrm{nsec}$ for fast frozen solutions measured at $-160^{\circ} \mathrm{C}$. The uncertainties are standard deviations as estimated by the computer. For comparison values for pure ice and HF are included. The uncertainties on the concentrations are estimated to be $\pm 20 \%$.

\begin{tabular}{lcc}
\hline \hline & $\begin{array}{c}\text { Concentration } \\
\text { (mole fraction) }\end{array}$ & $\begin{array}{c}I_{3} \pm \Delta I_{3} \\
(\%)\end{array}$ \\
\hline Pure ice & 0 & $1.5 \pm 0.5$ \\
$\mathrm{HF}$ & $10^{-4}$ & $52 \pm 2$ \\
$\mathrm{LiF}$ & $10^{-4}$ & $11.9 \pm 0.8$ \\
$\mathrm{NH}$ & $1.3 \times 10^{-4}$ & $6.5 \pm 0.7$ \\
$\mathrm{CsF}$ & $10^{-4}$ & $8.2 \pm 0.7$ \\
$\mathrm{HCl}$ & $10^{-4}$ & $31.2 \pm 0.6$ \\
$\mathrm{HCl}$ & $10^{-2}$ & $50.3 \pm 0.7$ \\
$\mathrm{HBr}$ & $10^{-4}$ & $37.2 \pm 0.8$ \\
$\mathrm{HI}$ & $10^{-4}$ & $23.3 \pm 1.4$ \\
$\mathrm{H} \mathrm{SO}_{4}$ & $1.1 \times 10^{-4}$ & $3.2 \pm 0.6$ \\
$\mathrm{NaCl}$ & $10^{-5}$ & $5.8 \pm 0.8$ \\
\hline \hline
\end{tabular}

times and relative intensities, as given in Table I. No such difference is found between the two solid compounds.

The viscosity of $\mathrm{D}_{2} \mathrm{O}$ is $20 \%$ larger than for $\mathrm{H}_{2} \mathrm{O} \cdot{ }^{18}$ If the bubble model applies for Ps in water, this may be related to a smaller amplitude of oscillation of the bubble volume, which in turn will produce a smaller pickoff annihilation rate. In ice the rigid structure will prevent formation of bubbles, and since no difference exists between the lattice parameters of $\mathrm{H}_{2} \mathrm{O}$ and $\mathrm{D}_{2} \mathrm{O}$, no difference in lifetime should be expected. The localization of Ps in a bubble in water may explain the broadening of the narrow component in angular correlation curves ${ }^{25}$ compared to low temperature ice, in which Ps is delocalized. ${ }^{7}$

An explanation of the differences in Ps-formation probabilities in ice and water and between liquid $\mathrm{H}_{2} \mathrm{O}$ and $\mathrm{D}_{2} \mathrm{O}$ will probably require a detailed knowledge of the slowing-down processes of positrons and Ps since a simple Ore gap model does not apply to the problem. No attempt to explain these processes will be carried out here.

It seems, however, to be a general feature of a liquid whose molecules contain hydrogen, that deuteration will decrease the Ps yield. ${ }^{19,26,27}$

One would expect that the components $\tau_{1}, I_{1}$ and $\tau_{2}, I_{2}$ in a spectrum for water could be associated with annihilation of para-Ps and of free positrons, respectively. This is not directly possible (Table I) since it would imply that $\tau_{1} \simeq 0.12 \mathrm{nsec}$ and $I_{1} \simeq I_{3} / 3$. The lifetime of free positrons in water is best found in an aqueous solution of a Ps oxidizer, such as $\mathrm{KMnO}_{4}$. The spectrum of this solution contains one single lifetime $\tau_{\text {free }}=$ $0.395 \pm 0.005$ nsec in both $\mathrm{H}_{2} \mathrm{O}$ and $\mathrm{D}_{2} \mathrm{O}$. As discussed earlier, a reason for the fact that the results for pure water (Table I) do not agree with expectations may be that the spectrum contains more than three lifetime components. That will be the case if more annihilation processes take place in water than considered above. Also an incomplete correction for annihilation in the source will have that effect. ${ }^{15}$ Actually an analysis with $\tau_{2}$ fixed at 0.40 nsec gives a reasonably good fit (within one standard deviation of the variance) and the parameters thus obtained are quite close to the expected ones.

\section{B. Pure Ice}

For pure ice at temperatures below $-100^{\circ} \mathrm{C}$ we may ascribe the three lifetime components in Fig. 4 to annihilation of ortho-Ps $\left(\tau_{3}, I_{3}\right.$, the basis of the analysis), free positrons $\left(\tau_{2}, I_{2}\right)$, and para-Ps $\left(\tau_{1}, I_{1}\right)$, respectively. The value $\tau_{2}=0.44$ nsec seems reasonable compared to the free positron lifetime $(0.40 \mathrm{nsec})$ in water, which is approximately $9 \%$ more dense than ice. The value $\tau_{1}=0.12 \mathrm{nsec}$ is in good agreement with the lifetime of free para-Ps (which beomes $0.105 \mathrm{nsec}$ when pickoff is taken into account). Furthermore, $I_{1}$ is fairly close to $\frac{1}{3} I_{3}$ (the dashed line). 


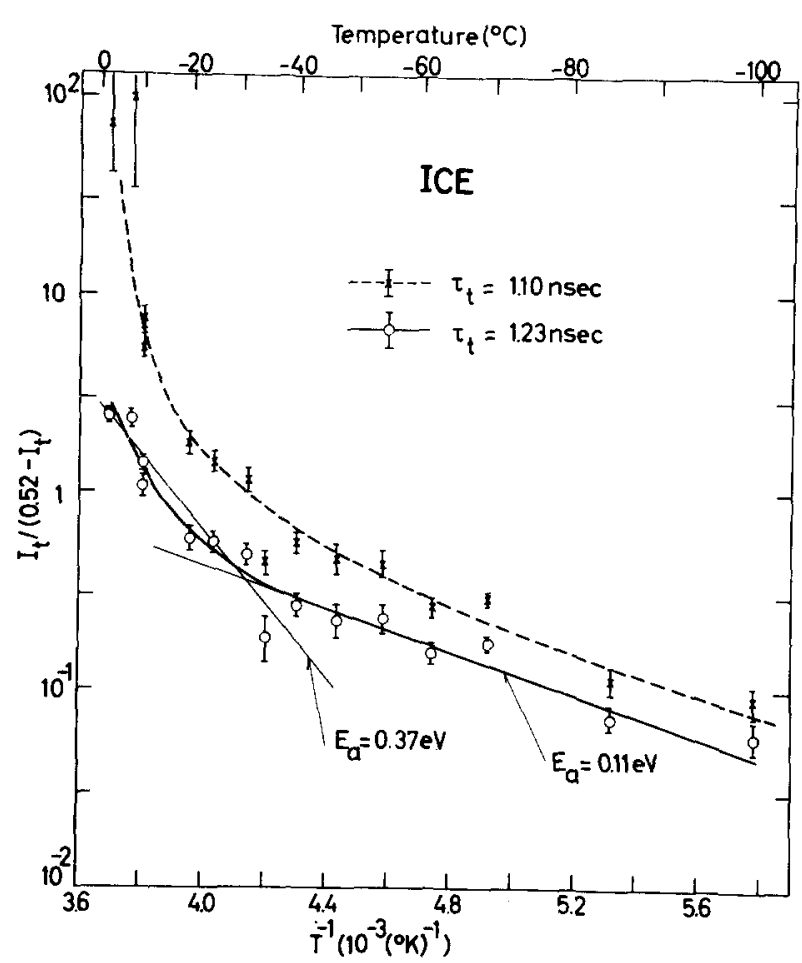

FIG. 7. The quantity $\left[I_{t} /\left(0.52-I_{t}\right)\right]$, which from a simple trapping model is proportional to the number of traps, plotted against $1 / T$ (Arrhenius plot) for two different $\tau_{t}$, the assumed ortho-Ps lifetime in the traps. $I_{t}$ is the relative intensity of the component with lifetime $\tau_{t}$. The slopes of the two straight lines estimate activation energies for possible temperature-created defects which trap ortho-Ps.

The rather steep rise of $\tau_{3}$ with temperature above $-100^{\circ} \mathrm{C}$ exceeds the variation expected from the free volume model. ${ }^{21}$ Ice has a free volume of around $66 \%$, and the cubical expansion of ice is about $1.6 \%$ when the temperature is raised from $-200^{\circ}$ to $0^{\circ} \mathrm{C}$. ${ }^{16}$ The effect of this temperature change, when calculated from a free volume model, ${ }^{21,24}$ will be an increase in the orthoPs lifetime of approximately $2 \%$. The expected variation is shown in Fig. 4. The dashed line was fitted to measurements at $-190^{\circ} \mathrm{C}$.

A more reasonable explanation of the large increase of the long lifetime is that trapping of ortho-Ps takes place in defects, the number of which increases with temperature. This explanation is supported by the temperature dependence of angular correlation curves for ice single crystals. ${ }^{10}$ With increasing temperature the area of the side peaks and the narrow central peak decreases and a component with a width of about $3.2 \mathrm{mrad}$ emerges. This strongly suggests that with increasing temperature an increasing number of para-Ps atoms becomes localized within a few angströms (i.e., becomes trapped). Hence, also ortho-Ps must be trapped, with a probability larger than for para-Ps, since the ortho-Ps lifetime is longer than that of para-Ps. This involves two characteristic decay rates of ortho-Ps. When defects are present, also single positrons, not bound in Ps, may be trapped in the way one has seen for metals. ${ }^{3}$ Hence it is likely that the decay of the positrons not forming $P_{s}$ will also be governed by two different rates, and the lifetime spectra for ice at higher temperatures may contain at least five components. Therefore, $\tau_{3}$ in Fig. 4 is only certain to be an average of the longest lifetimes in a spectrum (mainly due to ortho-Ps), and the peculiar behaviors of $\tau_{2}$ and $I_{1}$ may be caused by the mixing of the shorter lifetime components.

If the traps for ortho-Ps are a kind of temperaturecreated defects, the number of traps $N$ should depend upon temperature $T$ as

$$
N=N_{0} \exp \left(-E_{a} / k T\right),
$$

where $N_{0}$ is a constant, $E_{a}$ is the activation energy for creation of a defect, and $k$ is Boltzmann's constant. Experimentally the relative variation of the number of traps can be determined if the ortho-Ps lifetime in the traps, $\tau_{t}$, is known. Keeping one lifetime fixed at $\tau_{t}$ in the analysis of the spectra gives the relative intensity $I_{t}$ of this lifetime component. It is easy to show from Eq. (1) that the number of traps then will be proportional to $N^{\prime}=\left[I_{t} /\left(0.52-I_{t}\right)\right]$, where the total ortho-Ps yield is $52 \%$. Hence, a plot of $\log N^{\prime}$ against $1 / T$ (Arrhenius plot) will show a straight line if the above mentioned exponential dependence exists. From the slope of the line, $E_{a}$ can be determined.

This analysis (three terms) has been carried out for different fixed values of $\tau_{\ell}$, ranging from 1.1 to $1.7 \mathrm{nsec}$. The plot is shown in Fig. 7 for the values 1.1 and 1.23 nsec. (The value 1.23 nsec was taken from the results for frozen solutions of HF. However, since the nature of traps is probably different in the two cases, the lifetimes in the traps need not be the same. Actually, Fig. 1 suggests that $\tau_{t}>1.23 \mathrm{nsec}$ in pure ice.) The important point is that it is not possible to fit the points to a single straight line in a reasonable way whatever value is used for $\tau_{t}$. To determine the order of magnitude of the activation energies involved, the points for $\tau_{t}=1.23$ nsec was fitted with two straight lines. The associated energies are 0.11 and $0.37 \mathrm{eV}$. The same or slightly smaller activation energies are found for larger $\tau_{t}$ values.

The nonlinearity of the Arrhenius plot indicates that the increase of lifetime with temperature is not simply caused by trapping of ortho-Ps in one kind of thermally created defects in which it has one well-defined lifetime.

It is difficult to find a correlation between the different kinds of defects that are generally believed to exist in ice and the trapping of ortho-Ps. An estimated vacancy concentration at $-10^{\circ} \mathrm{C}$ is approximately $10^{-10}$ mole fraction, while the concentration of interstitials and ionic defects are about $10^{-11}$ mole fraction. ${ }^{16}$ Since it seems to be quite general that defect concentrations have to be of the order of parts per million to be detected by positrons or Ps (previous section and Refs. 3, $4,5,6$, and 28), these defect concentrations in ice are too small to act as efficient trapping centers. Also the 
effect of dislocations on Ps must be too small to be observed. A typical density is $10^{6} \mathrm{~cm}^{-2}$ which roughly speaking is equivalent to at most $10^{-9}$ mole fraction of point defects. It is likely that the number of dislocations is different for the various samples, in particular between the mono- and polycrystalline samples. The fact that the results are the same for the different ice samples shows that dislocations have no influence on the positron lifetimes. Neither has any annealing effect been observed, although of two subsequent measurements, the first was normally carried out at a high temperature, and the second at a lower one. The only defects of sufficient concentration are $\mathrm{L}$ and $\mathrm{D}$ defects. Ice consists of oxygen atoms connected tetrahedrally by hydrogen bonds. An $\mathrm{L}$ defect exists if the proton is missing in a bond, and a $\mathrm{D}$ defect exists if two protons are in the same bond. At $-10^{\circ} \mathrm{C}$ the concentration of these defects is about $10^{-6}$ mole fraction, and the energy required to form a pair of these defects is $0.68 \mathrm{eV} \cdot{ }^{16,18}$ The apparent activation energy for the formation of an $\mathrm{L}$ or a $\mathrm{D}$ defect will then be $\frac{1}{2} 0.68 \mathrm{eV}=$ $0.34 \mathrm{eV},{ }^{16}$ close to the $0.37 \mathrm{eV}$ estimated from Fig. 7 .

It should be noted, however, that the picture of $\mathrm{L}$ defects capturing $P_{s}$ is not quite consistent with our results for impurities in ice. The concentration of $L$ defects is generally believed to increase with $\mathrm{HF}$ doping and decrease with $\mathrm{NH}_{3}$ doping (and vice versa for $\mathrm{D}$ defects). However, both of these dopings tend to increase the long positron lifetime (see Fig. 5 and Table III), thus suggesting that neither $\mathrm{L}$ nor $\mathrm{D}$ defects are responsible for the trapping of Ps.

\section{Impurities in Ice}

The good agreement (except for very low $\mathrm{HF}$ concentrations) between the trapping model and the measurements on fast frozen HF solutions strongly supports the assumption that ortho-Ps gets trapped in defects created by $\mathrm{HF}$ in the ice matrix during fast freezing. By differential thermal analysis Vuillard has shown that a number of fast frozen solutions of acids, bases, and salts are in a glassy state at low temperatures and exhibit a glass transition at a well-defined temperature in the interval between -145 and $-110^{\circ} \mathrm{C}^{29} \mathrm{In}$ the light of this, our results may be interpreted as follows. By the fast freezing, an amorphous region of $\mathrm{H}_{2} \mathrm{O}$ molecules is formed about each impurity, the detailed constitution of which is not known (could be $\mathrm{F}^{-}, \mathrm{HF}, \mathrm{HF}_{2}^{-}$, or more complex molecules). The delocalized Ps atom can become trapped in the larger holes in the amorphous region and thus, due to more space, it obtains a longer lifetime. The reaction constant for the trapping of ortho-Ps, as calculated from the results of Fig. 5 is $6 \times 10^{12}$ liters $/$ mole. sec, which is about a hundred times more than the largest quenching constants known for aqueous solutions. The corresponding capture cross section is $22 \AA^{2}$, when the Ps atom is assumed to have thermal velocity. This large cross section might be interpreted in terms of delocalization of Ps.

When the amorphous regions are heated above approximately $-120^{\circ} \mathrm{C}$, an irreversible transition to a crystalline state is apparently taking place (Fig. 6). Due to the presence of the impurities, however, the lattice is distorted and will still give rise to trapping of Ps, but with smaller probability than for the amorphous regions.

We see from Fig. 5 that a characteristic lower defect concentration to be detected by positrons is $10^{-6}$ to $10^{-5}$ mole fraction in ice. As mentioned in the previous paragraph this seems to be quite generally the order of magnitude for the trapping center concentration necessary to get a measurable influence on positrons or positronium.

The influence on positron lifetimes by other acids and salts in fast frozen aqueous solutions, as investigated here (Table III), is smaller than that of HF although all of them most probably form glassy phases by fast freezing. ${ }^{29}$ The hydrogen halides show a quite strong influence, whereas a large molecule like $\mathrm{H}_{2} \mathrm{SO}_{4}$ has almost no effect. The results for the fluoride salts indicate that the presence of the $\mathrm{F}^{-}$ion in the solution before freezing does not necessarily lead to a large increase in lifetime. Furthermore, the results in Ref. 30 show that a glassy structure need not entail a lengthening of the ortho-Ps lifetime compared to crystalline ice. An additional fact is that the influence of $\mathrm{HCl}$ impurities on the angular correlation curve of ice is stronger than that of $\mathrm{HF}$ impurities. ${ }^{9}$

The present results and those discussed in Ref. 9 provide some knowledge about the influence of different impurities on the behavior of positrons and Ps in ice. However, more extensive investigations in the field of frozen aqueous solutions are necessary to get a detailed picture of the different annihilation processes.

\section{CONCLUSIONS}

The present work has been concentrated on an experimental investigation of positron lifetimes in the solid and liquid forms of light and heavy water and in frozen aqueous solutions. The temperature region which was investigated ranged from -190 to $+20^{\circ} \mathrm{C}$. All spectra were resolved into three lifetime components. The following major results were obtained.

Previously ${ }^{8}$ it was established that an abrupt change in ortho-Ps yield and lifetime takes place at the solidliquid phase transition for both light and heavy water. In the liquid the lifetime is longer and the yield smaller in heavy $(2.01 \pm 0.06 \mathrm{nsec}, 22.2 \pm 1.2 \%)$ than in light water $(1.86 \pm 0.04 \mathrm{nsec}, 26.9 \pm 1.0 \%)$ while in ice no difference is found. Also mono- and polycrystalline samples give identical results. In the temperature range from -190 to $-100^{\circ} \mathrm{C}$ essentially no change of the lifetime spectrum takes place as a function of temperature, in agreement with a free volume model. From 
$-100^{\circ} \mathrm{C}$ to the melting point the longest lifetime increased from $0.68 \pm 0.02$ to $1.12 \pm 0.02 \mathrm{nsec}$, its intensity in both intervals being $52 \%$. The increase in lifetime was ascribed to trapping of ortho-Ps in temperature-created defects although no clear-cut correlation with already known defects could be established. The large Ps yield of $70 \pm 3 \%$ found in ice is probably the biggest one observed in any substance.

In frozen aqueous solutions of $\mathrm{HF}$ trapping of $\mathrm{Ps}_{\mathrm{s}}$ was found to occur even for concentrations less than parts per million.

The measurements could be interpreted in terms of a very simple trapping theory, thus serving as a first clear demonstration of Ps trapping in defects. At $-160^{\circ} \mathrm{C}$ the ortho-Ps lifetime in the traps was $1.23 \mathrm{nsec}$. The very high reaction constant associated with the trapping process in fast frozen solutions (about hundred times larger than the highest values known from aqueous solutions) was ascribed to the delocalization of $\mathrm{Ps}$ in ice. An irreversible transition was observed about $-125^{\circ} \mathrm{C}$ by heating of a fast frozen $\mathrm{HF}$ solution of a concentration of one part per million. It was ascribed to a phase transition from an amorphous to a crystalline state of a region around each impurity. Several other frozen aqueous solutions were investigated, but none of them showed as strong an influence on the lifetime spectrum as HF did.

From ice physics point of view the most interesting problem raised by our work is: What is the nature of the defects which trap Ps (in particular in pure, but also in doped ice).

The present work, together with corresponding measurements by the angular correlation technique, will probably make ice the substance which has been investigated in most detail by positron annihilation. The results obtained for this solid may therefore (although ice in some respects is a rather special substance) serve as a starting point in the interpretation of measurements on other materials. To serve this aim better, however, more measurements on ice would be desirable. Apart from the measurements in progress (see the Introduction) they might comprise an extension of the temperature interval down to liquid helium temperature, lifetime magnetic quenching measurements, and angular correlation magnetic quenching experiments on monocrystals. Also a detailed determination of the temperature dependence of the $3 \gamma$-annihilation rate and the effect of irradiation could be desired.

\section{ACKNOWLEDGMENT}

We are grateful to Dr. G. Kvajić for supplying the ice single crystals.

${ }^{1}$ V. I. Goldanskii, At. Energy Rev. 6, 3 (1968).

${ }^{2}$ Positron Annihilation, edited by A. T. Stewart and L. O. Roellig (Academic, New York, 1967).

${ }^{3}$ B. Bergersen and M. J. Stott, Solid State Commun. 7, 1203 (1969).

${ }^{4}$ B. T. A. McKee, W. Triftshäuser, and A. T. Stewart, Phys. Rev, Letters 28, 358 (1972).

${ }^{5}$ W. Brandt, H. F. Waung, and P. W. Levy, Phys. Rev. Letters 26, 496 (1971).

${ }^{6}$ W. C. Mallard and F. H. Hsu, Phys. Letters 38 A, 164 (1972).

${ }^{7}$ O. Mogensen, G. Kvajić, M. Eldrup, and M. MiloševiéKvajic, Phys. Rev. B 4, 71 (1971).

${ }_{8}$ K. Petersen, M. Eldrup, and G. Trumpy, Phys. Letters 31 A, 109 (1970)

${ }^{\circ} \mathrm{M}$. Milošević-Kvajić, O. Mogensen, G. Kvajić, and $\mathbf{M}$. Eldrup, J. Chem. Phys. J. Chem. Phys. 56, 2567 (1972).

${ }^{10} \mathrm{O}$. Mogensen et al. (unpublished).

${ }^{11} \mathrm{~L}$. Smedskjaer and G. Trumpy (unpublished).

${ }_{12} \mathrm{P}$. Kirkegaard and M. Eldrup, Computer Phys. Commun. (to be published).

${ }^{13} \mathrm{~W}$. Brandt and I. Spirn, Phys. Rev. 142, 231 (1966).

${ }^{14}$ R. T. Wagner and F. L. Hereford, Phys. Rev. 99, 593 (1955).

${ }_{15}$ M. Eldrup, Ph.D. thesis, Risö Report No. 254, 1971.

${ }^{10} \mathrm{~N}$. H. Fletcher, The Chemical Physics of Ice (Cambridge U. P., London, 1970).

${ }_{17}$ Physics of Ice, edited by N. Riehl, B. Bullemer, and H. Engelhardt (Plenum, New York, 1969).

${ }_{18} \mathrm{D}$. Eisenberg and W. Kauzmann, The Structure and Properties of Water (Oxford U. P., London, 1969).

${ }^{19}$ S. Y. Chuang and S. J. Tao, Phys. Letters 33A, 56 (1970). ${ }^{20}$ H. S. Landes, S. Berko, and A. J. Zuchelli, Phys. Rev. 103, 828 (1956).

${ }^{21}$ W. Brandt, S. Berko, and W. W. Walker, Phys. Rev. 120, 1289 (1960).

${ }^{22}$ W. H. Holt, S. Y. Chuang, A. M. Cooper, and B. G. Hogg, J. Chem. Phys. 49, 5147 (1968).

${ }^{23}$ J. Lee and G. J. Celitans, J. Chem. Phys. 44, 2506 (1966).

${ }^{24}$ R. K. Wilson, P. O. Johnson, and R. Stump, Phys. Rev. 129,2091 (1963).

${ }^{25}$ P. Colombino, B. Fiscella, and L. Trossi, Nuovo Cimento 38, 707 (1965).

${ }^{26}$ P. R. Gray, C. F. Cook, and G. P. Sturm, J. Chem. Phys. 48, 1145 (1968).

${ }^{27}$ K. P. Singh, R. M. Singru, and C. N. R. Rao, J. Phys. B 4, 261 (1971).

${ }^{28}$ G. Coussot, Ph.D. thesis, Rapport C.E.A., R4138, 1971.

${ }^{20}$ G. Vuillard, Ann. Chim. (Paris) 2, 231 (1957).

${ }^{30} \mathrm{M}$. Eldrup, O. Mogensen, and L. Kevan, Chem. Phys. Letters 10, 379 (1971). 